\title{
Magnetized Anisotropic Dark Energy Models in Barber's Second Self-Creation Theory
}

\author{
D. D. Pawar' ${ }^{1}$ and Y. S. Solanke ${ }^{2}$ \\ ${ }^{1}$ School of Mathematical Sciences, Swami Ramanand Teerth Marathwada University, Vishnupuri, Nanded 431 606, India \\ ${ }^{2}$ Mungsaji Maharaj Mahavidyalay, Darwha, Yavatmal, India
}

Correspondence should be addressed to D. D. Pawar; dypawar@yahoo.com

Received 2 April 2014; Revised 14 July 2014; Accepted 23 July 2014; Published 24 August 2014

Academic Editor: George Siopsis

Copyright (C) 2014 D. D. Pawar and Y. S. Solanke. This is an open access article distributed under the Creative Commons Attribution License, which permits unrestricted use, distribution, and reproduction in any medium, provided the original work is properly cited. The publication of this article was funded by SCOAP ${ }^{3}$.

The present paper deals with Bianchi type IX cosmological model with magnetized anisotropic dark energy by using Barber's selfcreation theory. The energy momentum tensor consists of anisotropic fluid with EoS parameter $\omega$ and a uniform magnetic field of energy density $\rho_{B}$. In order to obtain the exact solution we have assumed that dark energy components and the components of magnetic field interact minimally and obey the law of conservation of energy momentum tensors. We have also used the special law of variation for the mean generalized Hubble parameter and power law relation between scalar field and scale factor. Some physical and kinematical properties of the models have been discussed.

\section{Introduction}

In an attempt to produce continuous creation theories, in 1982, Barber [1] has proposed two self-creation theories modifying the Brans and Dicke [2] and Brans [3] theory of gravitation and Einstein theory of general relativity. In 1987 Brans has pointed out that the first theory of Barber is not satisfactory because of violation of equivalence principle. According to Brans, Barber's first theory is not in disagreement with experimental observations and is also not consistent in general. His second self-creation theory is a modification of Einstein's general theory of gravitation to variable G-theory which predicts local effect and is within the observational limit. In his postulate, the gravitational coupling of the Einstein field equations is allowed to be a variable scalar on the space time so that this scalar couples to the trace of energy momentum tensors. Hence the field equations in Barber's second self-creation theory are

$$
\begin{aligned}
& \text { (i) } R_{i j}=\frac{1}{2} g_{i j} R=-\frac{8 \pi}{\varphi} T_{i j}, \\
& \text { (ii) } \square \varphi=\frac{8}{3} \pi \lambda T .
\end{aligned}
$$

Pimental [4], Sing and Deo [5], Soleng [6, 7], Reddy [8, 9], Maharaj and Beesham [10], Venkateswaru and Reddy $[11,12]$,
Shanti and Rao [13], Mohanti and Pradhan [14], and Pawar et al. $[15,16]$ are some of the authors who have investigated the various aspects of Barber's second self-creation theory by using various cosmological models. As we are unknown about the dark energy but it is necessary to explain. The recent observations from high red shift type Ia supernovae indicate the accelerated expansion of the universe which is confirmed by the combinations of the results from the large scale distribution of galaxies and the remarkable data on cosmic microwave background (CMB). According to Riess et al. [17, 18], Permutter et al. [19-21], and Spergel et al. [22, 23], all these observational results strongly imply the existence of extra components with negative pressure. Thus all these observational results are supporting the fact that universe is accelerating. According to Caldwell et al. [24, 25], these observational findings also suggest that there should be a transition of the universe from the earlier deceleration phase to the recent accelerating phase. Even though at present, recent observations supported that the universe is accelerating, but according to physicists there is no guarantee that it will do so forever. Moreover, some of the physicists come to the decision that the present acceleration of the universe may be followed by deceleration, even though universe may have oscillatory mode of expansion. In order to explain the cosmic 
acceleration of the universe, the cosmologists have proposed many candidates. The cosmological constant is one of the most obvious theoretical candidates for dark energy despite having two limitations, namely, fine tuning and cosmic coincidence problems Cohen et al. [26], Copeland et al [27] and Deo et al [28]. In addition to the cosmological constant, an alternative proposal for dark energy is dynamical dark energy scenario. The quintessence, phantom field, Caldwell [24], Ratra and Peebles [29], quintom Elizalde et al. [30], Chaplygin gas models Kamenshchik et al. [31], tachyon field Padmanabhan [32], holographic, Setare [33] are some of the examples of dark energy models. Recently, Pawar et al. [34, 35] studied Kantowski-Sachs cosmological models with constant EoS parameter in Barber's second self-creation theory and dark energy models in Brans-Dicke theory of gravitation.

An anisotropic cosmological model plays an important role in the large scale behavior of the universe. Many researchers working on cosmology by using relativistic cosmological models have not given proper reasons to believe in a regular expansion for the description of the early stages of the universe. Anisotropies in the $\mathrm{CMB}$ are related to small perturbation, superimposed on the perfectly smooth background, which are believed to seed formation of galaxies and large scale structure in the universe. There are some experimental data of CMR and theoretical arguments which support the existence of anisotropic universe (Chimento [36], Misner [37], Land and Maqueijo [38], Demianski [39], and Pawar et al. [40, 41]).

In the present paper we have used magnetic anisotropic Bianchi type IX space time. Many researchers are using Bianchi type IX space time because this space time model allows not only expansion but also rotation and shear. Bali and Dave [42] have investigated this model in general theory of relativity. Tyagi and Sharma [43] have used this model for perfect fluid distribution in general relativity. Rao and Sanyasiraju [44, 45] have studied Bianchi types VIII and IX models in zero mass scalar fields and self-creation cosmology. Pawar and Dagwal [46] investigated Bianchi type IX two fluids cosmological models in general relativity. Most of the models have been studied by considering perfect fluid but it is not sufficient to describe the dynamics of an accelerating universe. This motivates the researchers to consider the models of the universe filled with DE and magnetic field. In the present paper we have investigated Bianchi type IX magnetized anisotropic space time Barber's second selfcreation theory. We have obtained the exact solution of the field equations. The physical and geometrical properties of the models are discussed.

\section{Metric and Field Equations}

We consider Bianchi type IX metric in the form

$$
\begin{aligned}
d s^{2}= & -d t^{2}+a^{2}(t) d x^{2}+b^{2}(t) d y^{2} \\
& +\left(b^{2} \sin ^{2} y+a^{2} \cos ^{2} y\right) d z^{2}-2 a^{2} \cos y d x d z
\end{aligned}
$$

where $a$ and $b$ are the functions of cosmic time $t$.
The field equations in Baber's second self-creation theory are

$$
\begin{gathered}
G_{i j}=R_{i j}-\frac{1}{2} g_{i j} R=\frac{-8 \pi}{\varphi} T_{i j}, \\
\square \varphi=\varphi_{; k}^{, k}=\frac{8}{3} \pi \lambda T,
\end{gathered}
$$

where $\lambda$ is coupling constant and $\varphi$ is Barber's scalar.

We consider the energy momentum tensor for the magnetized anisotropic DE fluid

$$
\begin{aligned}
& T_{i}^{j}=\operatorname{dia}\left[T_{1}^{1}, T_{2}^{2}, T_{3}^{3}, T_{4}^{4}\right] \operatorname{dia}\left[-p_{x}+\rho_{B},-p_{y}-\rho_{B}, p_{z}-\rho_{B}, \rho+\rho_{B}\right] \\
&=\operatorname{dia}\left[-\omega_{x} \rho+\rho_{B},-\omega_{y} \rho-\rho_{B},-\omega_{z} \rho-\rho_{B}, \rho+\rho_{B}\right] \\
&=\operatorname{dia}\left[-(\omega+\delta) \rho+\rho_{B},-(\omega+\gamma) \rho-\rho_{B},\right. \\
&\left.\quad-(\omega+\gamma) \rho-\rho_{B},\left(\rho+\rho_{B}\right)\right],
\end{aligned}
$$

where $\omega_{x}=\omega+\delta, \omega_{y}=\omega+\gamma$, and $\omega_{z}=\omega+\gamma$ are the directional EoS parameters on $X-, Y$-, and $Z$-axes, respectively. $\rho$ is the energy density of the dark energy and $\rho_{B}$ is the energy density of the magnetic field.

In a comoving coordinate system Einstein's field (3) with (4) and (5) for the Bianchi type IX space time metric (2) subsequently leads to the following system of equations:

$$
\begin{gathered}
\frac{2 \ddot{b}}{b}+\frac{\dot{b}^{2}}{b^{2}}+\frac{1}{b^{2}}-\frac{3}{4} \frac{a^{2}}{b^{4}}=-\frac{8 \pi}{\varphi}\left[-(\omega+\delta) \rho+\rho_{B}\right], \\
\frac{\ddot{a}}{a}+\frac{\ddot{b}}{b}+\frac{\dot{a} \dot{b}}{a} \frac{a^{2}}{4 b^{4}}=-\frac{8 \pi}{\varphi}\left[-(\omega+\gamma) \rho-\rho_{B}\right], \\
\frac{\ddot{a}}{a}+\frac{\ddot{b}}{b}+\frac{\dot{a} \dot{b}}{a b}+\frac{a^{2}}{4 b^{4}}=-\frac{8 \pi}{\varphi}\left[-(\omega+\gamma) \rho-\rho_{B}\right], \\
\frac{2 \dot{a} \dot{b}}{a b}+\frac{\dot{b}^{2}}{b^{2}}-\frac{1}{4} \frac{a^{2}}{b^{4}}+\frac{1}{b^{2}}=-\frac{8 \pi}{\varphi}\left[\left(\rho+\rho_{B}\right)\right], \\
-\ddot{\varphi}-\left(\frac{\dot{a}}{a}+2 \frac{\dot{b}}{b}\right) \dot{\varphi}=\frac{8}{3} \pi \lambda(1-\delta-2 \gamma-3 \omega) \rho .
\end{gathered}
$$

Thus the above system of the equations reduces and takes the form

$$
\begin{gathered}
\frac{2 \ddot{b}}{b}+\frac{\dot{b}^{2}}{b^{2}}+\frac{1}{b^{2}}-\frac{3}{4} \frac{a^{2}}{b^{4}}=-\frac{8 \pi}{\varphi}\left[-(\omega+\delta) \rho+\rho_{B}\right], \\
\frac{\ddot{a}}{a}+\frac{\ddot{b}}{b}+\frac{\dot{a} \dot{b}}{a b}+\frac{a^{2}}{4 b^{4}}=-\frac{8 \pi}{\varphi}\left[-(\omega+\gamma) \rho-\rho_{B}\right], \\
\frac{2 \dot{a} \dot{b}}{a b}+\frac{\dot{b}^{2}}{b^{2}}-\frac{1}{4} \frac{a^{2}}{b^{4}}+\frac{1}{b^{2}}=-\frac{8 \pi}{\varphi}\left[\left(\rho+\rho_{B}\right)\right], \\
-\ddot{\varphi}-\left(\frac{\dot{a}}{a}+2 \frac{\dot{b}}{b}\right) \dot{\varphi}=\frac{8}{3} \pi \lambda(1-\delta-2 \gamma-3 \omega) \rho .
\end{gathered}
$$


By the law of conservation we have the Bianchi identity

$$
\begin{gathered}
\dot{\rho}+\left(\frac{\dot{a}}{a}+\frac{2 \dot{b}}{b}\right)(1+\omega) \rho+\left(\frac{\dot{a}}{a} \delta+\frac{2 \dot{b}}{b} \gamma\right) \rho \\
+\left(\rho_{B}+\frac{4 \dot{b}}{b} \rho_{B}\right)=0 .
\end{gathered}
$$

Here we assume that DE components and magnetic field interact minimally.

\section{Solutions of the Field Equations}

The above system of the equations (7) to (11) containing eight unknown variables $a, b, \rho, \rho_{B}, \omega, \gamma, \delta$, and $\varphi$ in five linearly independent equations. The first three variables are Einstein field equations whereas the fourth and fifth variables are Barber's scalar field equation and Bianchi identity, respectively. In order to solve this undetermined system three additional constraints are required.

As the DE component and magnetic field interact minimally, the Bianchi identity given by (11) can be split up into two separately additive conserved components, namely, the energy momentum tensors for the dark energy and energy momentum tensor for the magnetic field,

$$
G_{\mu ; v}^{v}=T_{\mu ; v}^{v}+T_{\mu ; v}^{(\beta) v}=0 .
$$

Thus the conservations of the energy momentum of the $\mathrm{DE}$ are given by

$$
T_{\mu ; v}^{v}=\dot{\rho}+(1+\omega) \rho\left(\frac{\dot{a}}{a}+\frac{2 \dot{b}}{b}\right)+\rho\left(\frac{\dot{a}}{a} \delta+\frac{2 \dot{b}}{b} \gamma\right)=0 .
$$

And the conservation of the energy momentum tensor of the magnetic field is given by

$$
T_{\mu ; v}^{(B) v}=\dot{\rho_{B}}+\frac{4 \dot{b}}{b} \rho_{B}=0 .
$$

After integration $(14) \Rightarrow$

$$
\rho_{B}=\frac{k_{1}}{b^{4}}
$$

where $k_{1}$ is the constant of integration. But conservation of the energy momentum of the dark energy can also be decomposed into two parts:

$$
T_{\mu ; v}^{v}=T_{\mu ; v}^{\prime v}+\tau_{\mu ; v}^{v}=0 .
$$

The last term in (13) is $\tau_{\mu ; v}^{v}$ which arises due to deviation from EoS parameter $\omega$ whereas the first two terms of (13) are $T_{\mu ; v}^{\prime v}$ which arise due to no deviation. But according to our assumption (Akarsu and Kilinc [47]) we have

$$
\begin{gathered}
T_{\mu ; v}^{\prime v}=\dot{\rho}+(1+\omega) \rho\left(\frac{\dot{a}}{a}+\frac{2 \dot{b}}{b}\right)=0, \\
\tau_{\mu ; v}^{v}=\rho\left(\frac{\dot{a}}{a} \delta+\frac{2 \dot{b}}{b} \gamma\right)=0 .
\end{gathered}
$$

According to (17) and (18) the behavior of the energy density of the DE component is controlled by the deviation free part $T^{\prime}$ of the EoS parameter $\omega$ of the DE, but this deviation does not affect energy density of the DE directly. It affects EoS parameter $\omega$. The choice of skewness parameters $(\delta, \gamma)$ is arbitrary; it may be constant or it may be the function of cosmic time. But since we are interested in physically variable models of the universe consistent with observations, the skewness parameters $\delta$ and $\gamma$ are assumed to be the functions of cosmic time. Here the skewness parameters $\delta$ and $\gamma$ are assumed to be a special dynamics on $x, y$, and $z$ axes consistent with (18). The dynamics of the skewness parameters $\delta(t)$ on the $x$-axis is assumed to be (by Akarsu and Kilinc)

$$
\delta(t)=N \frac{2}{3} \frac{\dot{b}}{b}\left(\frac{\dot{a}}{a}+\frac{2 \dot{b}}{b}\right) \frac{1}{\rho^{(d e)}} .
$$

The dynamics of the skewness parameter $\gamma(t)$ on $y$ - and $z$ axes is given by

$$
\gamma(t)=-N \frac{1}{3} \frac{\dot{a}}{a}\left(\frac{\dot{a}}{a}+\frac{2 \dot{b}}{b}\right) \frac{1}{\rho^{(d e)}} .
$$

As per our assumption (Berman [48]), the variations of mean generalized Hubble's parameter for the Bianchi type IX metric may be given by

$$
H=l\left(a b^{2}\right)^{-n / 3}
$$

where $l>0$ and $n \geq 0$ are constants. The spatial volume $V$ is given by

$$
V=R^{3}=a b^{2}
$$

where $R$ is average mean scalar factor. The mean generalized Hubble parameter $H$ for the Bianchi type IX metric may be given by

$$
\begin{aligned}
H & =\frac{\dot{R}}{R}=\frac{1}{3} \frac{\dot{V}}{V}=\frac{1}{3}\left(H_{1}+H_{2}+H_{3}\right) \\
& =\frac{1}{3}\left(\frac{\dot{a}}{a}+\frac{\dot{b}}{b}+\frac{\dot{b}}{b}\right)=\frac{1}{3}\left(\frac{\dot{a}}{a}+\frac{2 \dot{b}}{b}\right),
\end{aligned}
$$

where $H_{1}$ and $H_{2}=H_{3}$ are the directional Hubble parameters along $x$ - and $y=z$-axes, respectively. They are defined by

$$
H_{1}=H_{x}=\frac{\dot{a}}{a}, \quad H_{2}=H_{y}=\frac{\dot{b}}{b}, \quad\left(H_{y}=H_{z}\right) .
$$

On integration, equating (21) and (23) yields

$$
\begin{gathered}
R=\left(n l t+c_{1}\right)^{1 / n}, \quad n \neq 0, \\
R=c_{2} e^{l t}, \quad n=0 .
\end{gathered}
$$

Thus (21) gives two cosmological models of the universe depending on the values of $n$. When $n \neq 0$, we are getting power expansion model and when $n=0$ we are 
getting exponential expansion model. Further in view of the anisotropy of the space time in order to obtain determinate solution we have to assume two more constraints as

$$
a=R^{m}
$$

where $m$ is constant and the power law relation between average scale factor $R$ and the scalar field $\varphi$ (Johri and Desikan [49]) yields

$$
\varphi \propto R^{L} \Longrightarrow \varphi=c R^{L}
$$

where $L$ is any integer and $c$ is the constant of proportionality.

3.1. Power Expansion Model When $n \neq 0$. Average scale factor for this model by (25) is

$$
R=\left(n l t+c_{1}\right)^{1 / n} .
$$

Hence the scalar field $\varphi$ from (25) and (28) is

$$
\varphi=c\left(n l t+c_{1}\right)^{L / n} .
$$

Equations (25) and (27) give

$$
a=\left(n l t+c_{1}\right)^{m / n} .
$$

From (22), (25), and (31) we have

$$
b=\left(n l t+c_{1}\right)^{(3-m) / 2 n} .
$$

Equations (22) and (25) give the spatial volume as

$$
V=\left(n l t+c_{1}\right)^{3 / n}
$$

The directional Hubble parameters defined by (24) take the form

$$
\begin{gathered}
H_{1}=\frac{l}{\left(n l t+c_{1}\right)}, \\
H_{2}=H_{3}=\frac{(3-m) l}{2\left(n l t+c_{1}\right)} .
\end{gathered}
$$

Hence the mean generalized Hubble parameter defined in (23) takes the form

$$
H=\frac{l}{\left(n l t+c_{1}\right)} .
$$

The expansion scalar $\theta$, mean anisotropy parameter $A_{m}$, and shear scalar $\sigma$ are, respectively, given by

$$
\begin{gathered}
\theta=\frac{3 l}{\left(n l t+c_{1}\right)}, \\
A_{m}=\frac{\left(m^{2}-2 m+1\right)}{2}, \\
\sigma^{2}=\frac{9 l^{2}}{2\left(n l t+c_{1}\right)^{2}} .
\end{gathered}
$$

Using (31) and (32) Bianchi type IX cosmological model in (2) in Barber's second self-creation theory takes the form

$$
\begin{aligned}
d s^{2}= & -d t^{2}+\left(n l t+c_{1}\right)^{2 m / n} d x^{2}+\left(n l t+c_{1}\right)^{(3-m) / n} d y^{2} \\
& +\left[\left(n l t+c_{1}\right)^{(3-m) / n} \sin ^{2} y+\left(n l t+c_{1}\right)^{2 m / n} \cos ^{2} y\right] d z^{2} \\
& -2\left(n l t+c_{1}\right)^{2 m / n} \cos y d x d z .
\end{aligned}
$$

Equations (15) and (31) give the energy density of magnetic field as

$$
\rho_{B}=\frac{k_{1}}{\left(n l t+c_{1}\right)^{2(3-m) / n}} .
$$

Using (9) with (30), (31), (32), and (40) energy density of the $\mathrm{DE}$ is

$$
\begin{aligned}
\rho= & \frac{-k_{1}}{\left(n l t+c_{1}\right)^{2(3-m) / n}} \\
& -\frac{c}{8 \pi}\left\{\frac{3(m+1)(3-m) l^{2}}{4\left(n l t+c_{1}\right)^{(2 n-L) / n}}-\frac{1}{4\left(n l t+c_{1}\right)^{(2(3-m)-L) / n}}\right.
\end{aligned}
$$

$$
\left.+\frac{1}{\left(n l t+c_{1}\right)^{(3-m-L) / n}}\right\} \text {. }
$$

Using (19) with (31), (32), and (41) skewness parameter $\delta(t)$ takes the form

$$
\begin{gathered}
\delta(t)=\left(\frac{32 \pi N(3-m) l^{2}}{\left(n l t+c_{1}\right)^{L / n}}\right) \\
\times\left[-32 \pi k_{1}\left(n l t+c_{1}\right)^{(2(m+n-3)-L) / n}\right. \\
\quad-\left(9+6 m-3 m^{2}\right) c l^{2}+c\left(n l t+c_{1}\right)^{2(2 m+n-3) / n} \\
\left.-4 c\left(n l t+c_{1}\right)^{[2 n-(3-m-2 L)] / n}\right]^{-1}
\end{gathered}
$$

Using (20) with (31), (32), and (41) the skewness parameter $\gamma(t)$ takes the form

$$
\begin{aligned}
\gamma(t)= & \left(\frac{-32 \pi N m l^{2}}{\left(n l t+c_{1}\right)^{L / n}}\right) \\
\times & {\left[-32 \pi k_{1}\left(n l t+c_{1}\right)^{(2(m+n-3)-L) / n}\right.} \\
& -\left(9+6 m-3 m^{2}\right) c l^{2}+c\left(n l t+c_{1}\right)^{2(2 m+n-3) / n} \\
& \left.-4 c\left(n l t+c_{1}\right)^{[2 n-(3-m-2 L)] / n}\right]^{-1} .
\end{aligned}
$$


Using (17) with (31), (32), and (41) the EoS parameter of the model is

$$
\begin{aligned}
\omega(t)=(2 m-3) k_{1} l\left(n l t+c_{1}\right)^{(2 m-n-6) / n} \\
+\frac{c}{8 \pi}\left[\frac{l^{3}(m+1)(3-m)(9+3 L-6 n)}{4}\right. \\
\left.\quad \times\left(n l t+c_{1}\right)^{(L-3 n) / n}\right] \\
+\frac{c}{8 \pi}\left[-\frac{(L+2 m-3) l}{4}\left(n l t+c_{1}\right)^{(2 m+L-n-6) / n}\right. \\
\left.+(L+m) l\left(n l t+c_{1}\right)^{(L+m-3-n)}\right] .
\end{aligned}
$$

3.1.1. Physical Behavior of the Model When $n \neq 0$. In this model the direction the directional Hubble parameter, mean generalized Hubble parameters, expansion scalar, Barber's scalar field, and shear scalar all are the functions of the cosmic time except the mean anisotropy parameter $A_{m}$. It is constant throughout the universe. All the above parameters vanish when cosmic time is infinite, whereas these parameters are finite when time is zero except the directional Hubble parameter $\mathrm{H}_{2}$. It follows the above property provided $m \neq 3$. The spatial volume of this model is also a function of cosmic time and it increases with increase in time. That is, the model is expanding with time. The energy density of the model is constant when cosmic time zero $m \neq 3$ but when $m=3$ the energy density of the magnetic field is $k_{1}$ and it vanishes when $t$ is infinite. The energy density of the $\mathrm{DE}$ is constant when cosmic time is zero and it tends to zero when cosmic time tends to infinite when $m \neq 3$ but when $m=3(L, n>0)$ the energy density of the DE is $-k_{1}+(3 c / 32 \pi)\left(n l t+c_{1}\right)^{L / n}$. Thus in this case the energy density of DE increases with increase in cosmic time and when $t=0$ energy density of $\mathrm{DE}$ is $-k_{1}+(3 c / 32 \pi) c_{1}^{L / n}=$ constant. Similarly the skewness parameters $\delta(t), \gamma(t)$ as well as EoS parameter $\omega(t)$ are the functions of cosmic time $t$. Also $\lim _{t \rightarrow \infty}(\sigma / \theta)^{2} \neq 0$. Hence this model does not approach the isotropy.

We now extend our work to investigate the consistency of model (39) with the observational parameters from the point of astrophysical phenomena.

(1) Look-Back Time Redshift. The look-back time $t_{L}$ is defined as the difference between the present age of the universe $t_{0}$ and the age of the universe $t$ when a particular light ray at redshift $z$ was emitted.

The average scale factor of the universe $R$ for a given redshift $z$ is related to the present scale factor $R_{0}$ by the relation $1+z=R_{0} / R$.

Equations (25) and (35) give

$$
H_{0}\left(t_{0}-t\right)=\frac{1}{n}\left[1-(1+z)^{-n}\right] .
$$

After little manipulation we get

$$
H_{0}\left(t_{0}-t\right)=z-\frac{(n-1)}{2} z^{2}+\cdots .
$$

Taking limit $z \rightarrow \infty$ in (45) the present age of the universe is

$$
H_{0}\left(t_{0}-t\right)=\frac{1}{n}[1-0] \Longrightarrow t_{0}=\frac{1}{n H_{0}}=\frac{H_{0}^{-1}}{n}, \quad n \neq 0,
$$

where $H_{0}$ is the present value of the Hubble parameter.

(2) Luminosity Distance Redshift. The luminosity distance $d_{L}$ of a light source is defined as

$$
d_{L}=R_{0} r_{1}(z)(1+z),
$$

where the radial coordinate distance $r_{1}(z)$ of the object at light emission is

$$
r_{1}(z)=\int_{t_{1}}^{t_{0}} \frac{d t}{R}=\frac{H_{0}^{-1} R_{0}^{-1}}{(n-1)}\left[1-(1+z)^{1-n}\right] .
$$

Solving (48) and (49) we get

$$
H_{0} d_{L}=\frac{(1+z)}{(n-1)}\left[1-(1+z)^{(1-n)}\right] .
$$

3.2. Exponential Expansion Model When $n=0$. Average scale factor for this model by (26) is

$$
R=c_{2} e^{l t} .
$$

Thus from (26) and (28) we have the scalar field as

$$
\varphi=c c_{2}^{L} e^{(L l t)} .
$$

Equations (26) and (27) $\Rightarrow$

$$
a=c_{2}^{m} e^{(m l t)} .
$$

Similarly (22), (26), and (53) $\Rightarrow$

$$
b=c_{2}^{(3-m) / 2} e^{((3-m) / 2) l t}, \quad m \neq 3 .
$$

From (22) and (26) the spatial volume of this model is

$$
V=c_{2}^{3} e^{(3 l t)} .
$$

The directional Hubble parameters $H_{1}, H_{2}=H_{3}$ are given by

$$
\begin{gathered}
H_{1}=m l, \\
H_{2}=H_{3}=\frac{(3-m) l}{2} .
\end{gathered}
$$

Thus the mean generalized parameters defined in (23) take the form

$$
H=l=\text { constant } .
$$

In this case, expansion scalar $\theta$, mean anisotropy parameter $A_{m}$, and shear scalar are, respectively, given by

$$
\begin{gathered}
\theta=3 l, \\
A_{m}=\frac{1}{2}\left(m^{2}-2 m+1\right), \\
\sigma^{2}=\frac{9 l^{2}}{2} .
\end{gathered}
$$


Using (53) and (54) Bianchi type IX cosmological model in (2) in Barber's second self-creation theory takes the form

$$
\begin{aligned}
d s^{2}= & -d t^{2}+c_{2}^{2 m} e^{(2 m l t)} d x^{2}+c_{2}^{(3-m)} e^{(3-m) l t} d y^{2} \\
& +\left[c_{2}^{(3-m)} e^{(3-m) l t} \sin ^{2} y+c_{2}^{2 m} e^{(2 m l t)} \cos ^{2} y\right] d z^{2} \\
& -2 c_{2}^{2 m} e^{(2 m l t)} \cos y d x d z .
\end{aligned}
$$

In this case (15) and (54) give the energy density of the magnetic field

$$
\rho_{B}=\frac{k_{1}}{c_{2}^{2(3-m)} e^{2(3-m) l t}} .
$$

Using (9) with (52), (53), (54), and (61) gives the energy density of the DE

$$
\begin{gathered}
\rho=-\frac{k_{1}}{c_{2}^{2(3-m)}} e^{-2(3-m) l t} \\
-\frac{c c_{2}^{L}}{8 \pi}\left\{\frac{(9+6 m-3 m)^{2} l^{2} e^{(L l t)}}{4}-\frac{c_{2}^{(4 m-6)}}{4} e^{(4 m+L-6) l t}\right. \\
\left.+c_{2}^{(m-3)} e^{(L+m-3) l t}\right\} .
\end{gathered}
$$

Equation (19) with (53), (54), and (62) gives the skewness parameter $\delta(t)$ as

$$
\begin{aligned}
\delta(t)=\left(32 \pi N(3-m) l^{2}\right) \\
\times\left(-32 \pi k_{1}\left[c_{2} e^{-l t}\right]^{2(3-m)}-\left(9+6 m-3 m^{2}\right) c c_{2}^{L} l^{2} e^{(L l t)}\right. \\
\left.\quad+c\left[c_{2} e^{l t}\right]^{(4 m+L-6)}-4 C\left[c_{2} e^{l t}\right]^{(L+m-3)}\right)^{-1}
\end{aligned}
$$

and similarly (20) with (53), (54), and (62) gives the skewness parameter $\gamma(t)$

$$
\begin{aligned}
\gamma(t)=( & \left.-32 \pi N m l^{2}\right) \\
\times & \left\{-32 \pi k_{1}\left[c_{2} e^{l t}\right]^{2(m-3)}-\left(9+6 m-3 m^{2}\right) c c_{2}^{L} l^{2} e^{(L l t)}\right. \\
+ & \left.c\left[c_{2} e^{l t}\right]^{(4 m+L-6)}-4 c\left[c_{2} e^{l t}\right]^{(m+L-3)}\right\}^{-1} .
\end{aligned}
$$

Equation (17) with (53), (54), and (62) gives the EoS parameter $\omega(t)$ as

$$
\begin{aligned}
\omega(t)=(2 m-3) & k_{1} l\left[c_{2} e^{l t}\right]^{2(m-3)} \\
+\frac{c c_{2}^{L}}{8 \pi}[ & \frac{(3+L) l^{3}\left(9+6 m-3 m^{2}\right) e^{(L l t)}}{4} \\
& -\frac{(4 m+L-3) l c_{2}^{2(2 m-3)} e^{(4 m+L-6) l t}}{4} \\
& \left.+(L+m) l c_{2}^{(m-3)} e^{(L+m-3) l t}\right] .
\end{aligned}
$$

3.2.1. The Physical Behavior of the Model When $n=0$. For this model average scale factor $R=c_{2} e^{l t}$ and the scalar field $\varphi=c c_{2}^{L} e^{L l t}$. This model is nonsingular since the exponential function never vanishes. Thus this model does not have any physical singularity. For this model the directional Hubble parameters as well as mean generalized Hubble parameter $H$ are constant throughout the evolution of the universe. Hence, $d H / d t=0$. This implies that the greater the value of Hubble parameter, the faster the rate of expansion of the universe. Hence, this model may represent inflationary era in the early stages of the universe and the very late time of the universe. The spatial volume of the universe is finite when cosmic time is zero and expands exponentially as $t$ increases and becomes infinite when $t=\infty$ which indicates that universe starts its expansion with zero volume from finite past. In this case the expansion scalar $\theta$ and the mean anisotropy parameter $A_{m}$ are constants throughout the evolution of the universe but mean anisotropy parameter is exactly the same as the previous model. The energy density of the magnetic field is $\rho_{B}$ $=k_{1} / c_{2}^{2(3-m)}=$ constant when time $t=0$ and $m \neq 3$ but when $m=3$ it is equal to constant $k_{1}$. The density of the magnetic field vanishes when the cosmic time is infinite. Energy density of the DE is some finite number when cosmic time is zero and it increases with increase in time and it becomes infinite when time is infinite. The skewness parameters $\delta(t), \gamma(t)$ as well as EoS parameters $\omega(t)$ are the functions of cosmic time $t$. In this model also $\lim _{t \rightarrow \infty}(\sigma / \theta)^{2} \neq 0$. Hence this model does not approach the isotropy.

In this case also we extend our work to investigate the consistency of model (57) with the observational parameters from the point of astrophysical phenomena.

(1) Look-Back Time Redshift. From (26) and (53) we have

$$
H\left(t_{0}-t\right)=\log (1+z) \text {. }
$$

Thus for small $z$ we have

$$
\begin{array}{r}
H_{0}\left(t_{0}-t\right)=\log (1+z)=\left[z-\frac{z^{2}}{2}+\cdots\right], \\
\text { where } t=t_{0}-\frac{\log (1+z)}{H} .
\end{array}
$$


(2) Luminosity Distance Redshift. The luminosity distance of light source is given by

$$
H_{0} d_{L}=z(1+z) \Longrightarrow d_{L}=\frac{z(1+z)}{H}
$$

The above equation shows that the luminosity distance increases faster with the redshift when $n=0$.

\section{Conclusion}

In the present paper, we have discussed magnetized Bianchi type IX cosmological model in Barber's second self-creation theory. We have studied the minimal interaction between magnetic field and DE which will help us in detecting DE. We have obtained the exact solution of the Bianchi type IX cosmological model. It is observed that in both models EoS parameter is a function of cosmic time which supported the recent observations. The interesting and remarkable observation of the present paper is that the mean anisotropy parameter $A_{m}$ for both models is the same and is constant. Also $\lim _{t \rightarrow \infty}(\sigma / \theta)^{2} \neq 0$ for both models. Hence both models do not approach the isotropy.

We have also studied the two astrophysical phenomena such as look-back time redshift and luminosity distance redshift for both models and observed that such models are compatible with recent observations because from the point of recent astronomical measurements obtained by Pawar et al. [15, 16], Riess et al. [17, 18], and Permutter et al. [19] the SN (supernovae) Hubble diagram derived from the observations of hundreds of SNe detected over the border range of redshifts has amply demonstrated that the universe is expanding with acceleration. Further confirmation for the accelerating universe came from the recent observations of Ia type of SNe studied by Pawar et al. [16], Tonry et al. [50], and Clocchiatti et al. [51]. Within the general relativity, in order to explain the accelerated expansion of the universe, a nearly smooth form of energy of large negative pressure is inevitable. Confirmation by Bennett et al. [52] regarding the filling-in of the universe by a dominated component of energy with large negative pressure, dubbed as dark energy, was provided by the measurements of cosmic microwave background (CMB) and large scale structure (LSS) studied by Tegmark et al. [53]. This form of energy is believed to account for about $75 \%$ of the universe. The current observational data obtained by Frieman et al. [54] provide strong evidence regarding the existence of dark energy and further demonstrate that the fractional of critical that is being contributed by the dark energy may go as high as $0.76 \backslash \mathrm{pm} 0.02$.

Our present model is noninteracting Bianchi type IX cosmological model which cannot resolve the coincidence problem since it predicts inclusive system in which magnetic energy density decreases faster (which is inversely proportional to scale factor), while density of the dark energy either remains constant or increases. The interacting models play an important role in the study of coincidence problem $[55,56]$.

\section{Conflict of Interests}

The authors declare that there is no conflict of interests regarding the publication of this paper.

\section{Acknowledgment}

The authors would like thank the referee for invaluable suggestions.

\section{References}

[1] G. A. Barber, "On two "self-creation" cosmologies," General Relativity and Gravitation, vol. 14, no. 2, pp. 117-136, 1982.

[2] C. Brans and R. H. Dicke, "Mach's principle and a relativistic theory of gravitation," Physical Review, vol. 124, no. 3, pp. 925$935,1961$.

[3] C. H. Brans, "Consistency of field equations in "self-creation" cosmologies," General Relativity and Gravitation, vol. 19, no. 9, pp. 949-952, 1987.

[4] L. O. Pinental, "Exact self-creation cosmological solutions," Astrophysics and Space Science, vol. 116, pp. 395-399, 1985.

[5] R. T. Sing and S. Deo, "Zero-mass scalar interactions in the Robertson-Walker Universe," Acta Physica Hungarica, vol. 59, no. 3-4, pp. 321-325, 1986.

[6] H. H. Soleng, "Cosmic shear in inflationary models of Bianchi types VIII and IX," Astrophysics and Space Science, vol. 137, pp. 373-384, 1987.

[7] H. H. Soleng, "Self-creation cosmological solutions," Astrophysics and Space Science, vol. 139, no. 1, pp. 13-19, 1987.

[8] D. R. K. Reddy, "Bianchi type-I vacuum model in self-creation cosmology," Astrophysics and Space Science, vol. 132, no. 2, pp. 401-403, 1987.

[9] D. R. K. Reddy, "Vacuum friedmann model in self-creation cosmology," Astrophysics and Space Science, vol. 133, no. 1, pp. 189-191, 1987.

[10] S. D. Maharaj and A. Beesham, "Open and closed RobertsonWalker-type Lyttleton-Bondi universes," Astrophysics and Space Science, vol. 140, no. 1, pp. 33-37, 1988.

[11] R. Venkateswaru and D. R. K. Reddy, "Bianchi type-V radiating model in self creation cosmology," Astrophysics and Space Science, vol. 151, p. 353, 1989.

[12] R. Venkateswaru and D. R. K. Reddy, Astrophysics and Space Science, 152,337, 1989.

[13] K. Shanti and V. U. M. Rao, "Bianchi type-II and III models in self-creation cosmology," Astrophysics and Space Science, vol. 179, no. 1, pp. 147-153, 1991.

[14] G. Mohanti and B. D. Pradhan, "Cosmological mesonic viscous fluid model," International Journal of Theoretical Physics, vol. 1, article 31, 1992.

[15] D. D. Pawar, S. N. Bayaskar, and V. R. Patil, "Plane symmetric cosmological model with thick domain walls in Brans-Dicke theory of gravitation," Bulgarian Journal of Physics, vol. 36, pp. 68-75, 2009.

[16] D. D. Pawar, S. N. Bayaskar, and A. G. Deshmukh, "String cosmological model in presence of massless scalar field in modified theory of general relativity," Romanian Reports of Physics, vol. 56, no. 5-6, pp. 842-848, 2011.

[17] A. G. Riess, A. V. Filippenko, P. Challis et al., "Observational evidence from supernovae for an accelerating universe and 
a cosmological constant," The Astronomical Journal, vol. 116, article 1009, 1998.

[18] A. G. Riess, L.-G. Strolger, J. Tonry et al., “Type Ia supernova discoveries at $z>1$ from the Hubble Space Telescope: evidence for past deceleration and constraints on dark energy evolution," The Astrophysical Journal, vol. 607, pp. 665-687, 2004.

[19] S. Permutter, S. Gabi, G. Goldhaber et al., "Measurements of the cosmological parameters omega and lambda from the first 7 supernovae at $z \geq 0.35$," The Astrophysical Journal, vol. 483, p. 565, 1997.

[20] S. J. Permutter, G. Aldering, M. Della Valle et al., "Discovery of a supernova explosion at half the age of the Universe," Nature, vol. 391, pp. 51-54, 1998.

[21] S. J. Permutter, G. Aldering, G. Goldhaber et al., "Measurements of $\Omega$ and $\Lambda$ from 42 High-Redshift Supernovae," The Astrophysical Journal, vol. 517, no. 2, p. 565, 1999.

[22] D. N. Spergel, L. Verde, H. V. Peiris et al., "First-year Wilkinson Microwave Anisotropy Probe (WMAP) observations: determination of cosmological parameters," The Astrophysical Journal Supplement Series, vol. 148, no. 1, p. 175, 2003.

[23] D. N. Spergel, R. Bean, O. Doré et al., “Three-year Wilkinson Microwave Anisotropy Probe (WMAP) observations: implications for cosmology," The Astrophysical Journal Supplement Series, vol. 170, no. 2, article 377, 2007.

[24] R. R. Caldwell, “Cosmic Microwave Background and Supernova constraints on quintessence: concordance regions and target models," Physical Review D, vol. 69, 2004.

[25] R. R. Caldwell, "Sudden gravitational transition," Physical Review D, vol. 73, 2006.

[26] A. G. Cohen, D. B. Kaplan, and A. E. Nelson, "Effective field theory, black holes, and the cosmological constant," Physical Review Letters, vol. 82, no. 25, pp. 4971-4974, 1999.

[27] E. J. Copeland, M. Sami, and S. Tsujikawa, "Dynamics of dark energy," International Journal of Modern Physics, vol. 15, no. 11, pp. 1753-1935, 2006.

[28] A. Deo, J. S. Alcaniz, and D. Jain, "Cosmological consequences of a chaplygin gas dark energy," Physical Review D, vol. 67, Article ID 023515, 2004.

[29] B. Ratra and J. Peebles, "Fractal model for disordered magnets," Physical Review D, vol. 37, p. 321, 1988.

[30] R. R. Caldwell, "A phantom menace? Cosmological consequences of a dark energy component with super-negative equation of state," Physics Letters B, vol. 545, no. 1-2, pp. 23-29, 2002.

[31] E. Elizalde, S. Nojiri, and S. D. Odinstov, "Late-time cosmology in a (phantom) scalar-tensor theory: dark energy and the cosmic speed-up," Physical Review D, vol. 70, no. 4, Article ID 043539, 2004.

[32] A. Kamenshchik, U. Moschella, and V. Pasquier, "An alternative to quintessence," Physics Letters B, vol. 511, no. 2-4, pp. 265-268, 2001.

[33] T. Padmanabhan, "Accelerated expansion of the universe driven by tachyonic matter," Physical Review D, vol. 66, Article ID 021301, 2002.

[34] D. D. Pawar, Y. S. Solanke, and S. N. Bayaskar, "Kantowski-sachs cosmological models with constat EoS parameter in Barber's Second Self Creation Theory," Prespacetime Journal, vol. 5, no. 2, pp. 60-68, 2014.

[35] D. D. Pawar and Y. S. Solanke, "Exact kantowski-sachs anisotropic dark energy cosmological models in brans dicke theory of gravitation," International Journal of Theoretical Physics, vol. 53, no. 9, pp. 3052-3065, 2014.

[36] L. P. Chimento, "Extended tachyon field, Chaplygin gas, and solvable $k$-essence cosmologies," Physical Review D, vol. 69, no. 12, Article ID 123517, 10 pages, 2004.

[37] C. W. Misner, "The isotropy of the universe," The Astrophysical Journal, vol. 151, p. 431, 1968.

[38] K. Land and J. Maqueijo, "Examination of evidence for a preferred axis in the cosmic radiation anisotropy," Physical Review Letters, vol. 95, Article ID 071301, 2005.

[39] M. Demianski, "Tilted plane symmetric magnetized cosmological models," Physical Review D, vol. 46, no. 4, pp. 1391-1398, 1992.

[40] D. D. Pawar, V. D. Dagwal, and Y. S. Solanke, "Tilted plane symmetric magnetized cosmological models," Prespacetime Journal, vol. 5, no. 2, pp. 368-377, 2014.

[41] D. D. Pawar and V. J. Dagwal, "Bianchi type IX string cosmological model in general relativity," International Journal of Theoretical Physics, vol. 53, no. 7, pp. 2441-2450, 2014.

[42] R. Bali and S. Dave, "Bianchi type IX string cosmological model for perfect fluid distribution in general relativity," Pramana: Journal of Physics, vol. 56, p. 4, 2001.

[43] A. Tyagi and K. Sharma, "Bianchi type IX string cosmological model for perfect fluid distribution in general relativity," Chinese Physics Letters, vol. 27, no. 7, Article ID 079801, 2010.

[44] V. U. M. Rao and Y. V. S. S. Sanyasiraju, "Exact Bianchi-type VIII and IX models in the presence of zero-mass scalar fields," Astrophysics and Space Science, vol. 187, no. 1, pp. 113-117, 1992.

[45] D. D. Pawar and V. J. Dagwal, "Bianchi type IX two fluids cosmological models in general relativity," International Journal of Scientific and Engineering Research, vol. 4, no. 4, pp. 689-693, 2013.

[46] D. D. Pawar and V. J. Dagwal, "Bianchi type IX two fluids cosmological models in general relativity," International Journal of Scientific and Engineering Research, vol. 4, pp. 689-693, 2013.

[47] O. Akarsu and C. B. Kilinc, "LRS Bianchi type I models with anisotropic dark energy and constant deceleration parameter," General Relativity and Gravitation, vol. 42, pp. 119-140, 2010.

[48] M. S. Berman, "A special law of variation for Hubble's parameter," Il Nuovo Cimento B, vol. 74, pp. 182-186, 1983.

[49] V. B. Johri and K. Desikan, "Cosmological models with constant deceleration parameter in Brans-Dicke theory," General Relativity and Gravitation, vol. 26, no. 12, pp. 1217-1232, 1994.

[50] J. L. Tonry, B. P. Schmidt, B. Barris et al., "Cosmological results from high-z supernovae," The Astrophysical Journal, vol. 594, p. 1, 2003.

[51] A. Clocchiatti, B. P. Schmidt, and A. V. Filippenko, "Hubble Space Telescope and Ground-based Observations of Type Ia Supernovae at Redshift 0.5: Cosmological Implications," The Astrophysical Journal, vol. 642, no. 1, 2006.

[52] C. L. Bennett, R. S. Hill, G. Hinshaw et al., "First-year Wilkinson Microwave Anisotropy Probe (WMAP) observations: foreground emission," The Astrophysical Journal Supplement Series, vol. 148, no. 1, pp. 97-117, 2003.

[53] M. Tegmark, M. R. Blanton, M. A. Strauss et al., "The threedimensional power spectrum of galaxies from the sloan digital sky survey," The Astrophysical Journal, vol. 606, no. 2, article 702, 2004.

[54] J. Frieman, M. Turner, and D. Huterer, "Dark energy and the accelerating universe," Annual Review of Astronomy and Astrophysics, vol. 46, pp. 385-432, 2008. 
[55] S. del Campo, C. Fabris, R. Herrera, and W. Zimdahl, "Holographic dark-energy models," Physical Review D, vol. 83, Article ID 123006, 2011.

[56] S. del Campo, R. Herrera, and D. Pavon, "Interacting models may be key to solve the cosmic coincidence problem," Journal of Cosmology and Astroparticle Physics, vol. 2009, 2009. 

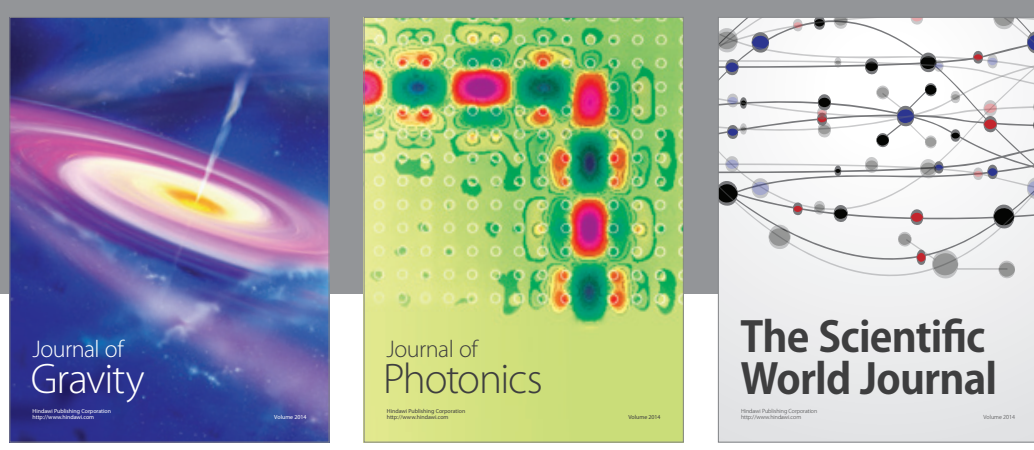

The Scientific World Journal
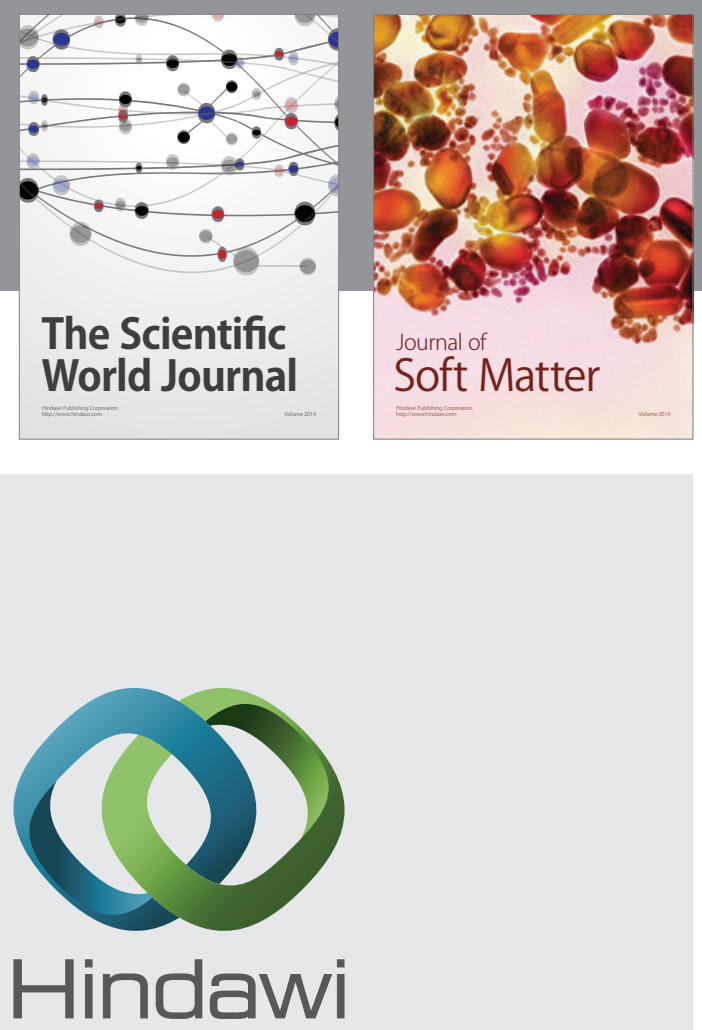

Submit your manuscripts at

http://www.hindawi.com

nternational Journal of

Statistical Mechanics
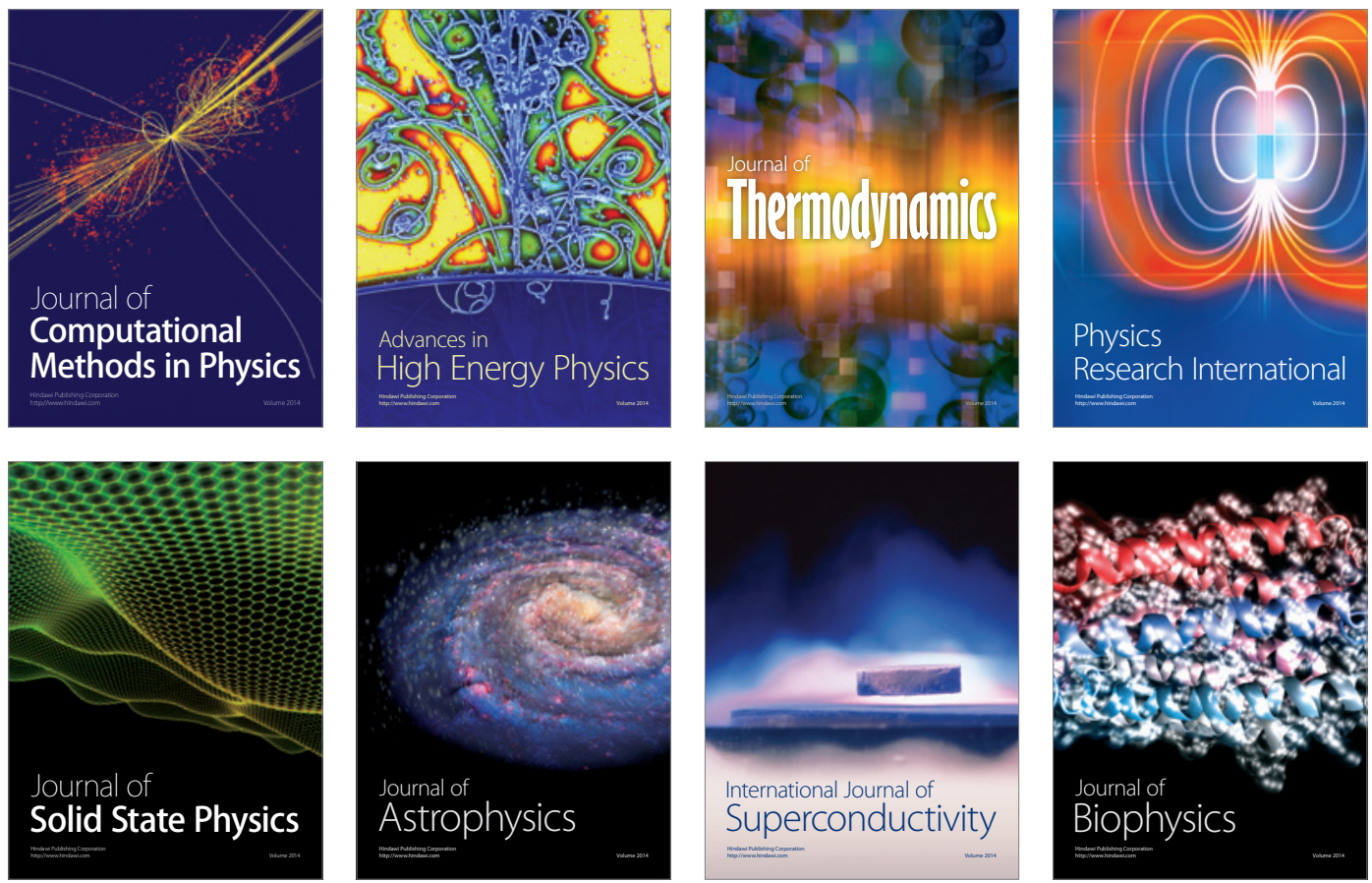
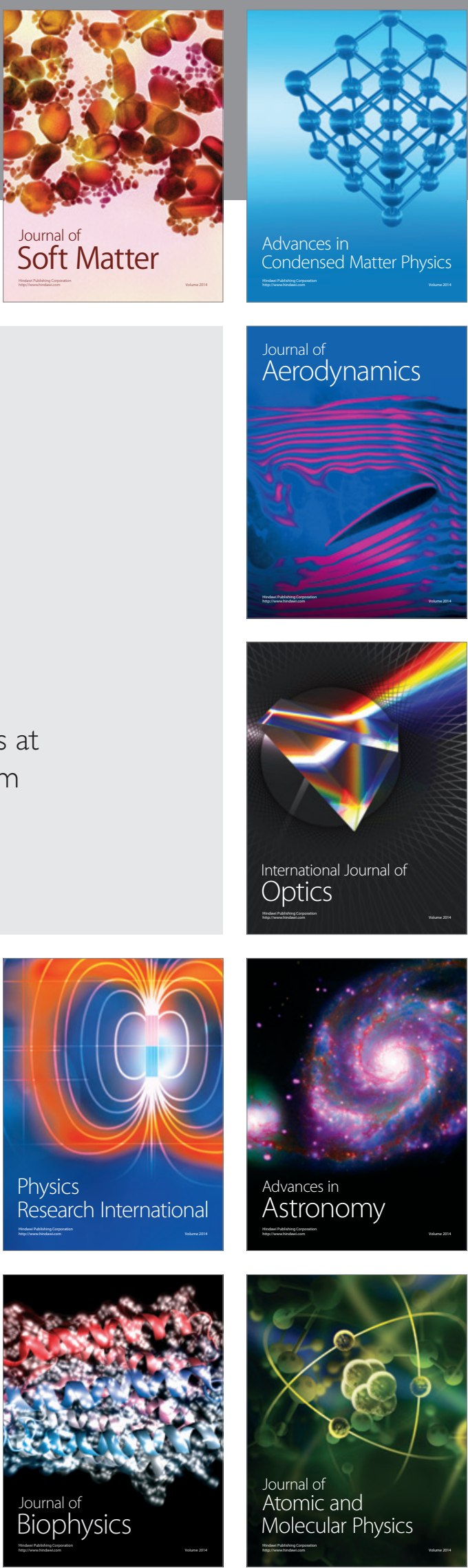